\title{
Oncogenic role of long non-coding RNA SNHG12 in gastric cancer cells by targeting miR-16
}

\author{
GUODONG ZHAO, SUINENG WANG, XIANWEN LIANG, CHUNFANG WANG and BO PENG \\ Department of Gastrointestinal Surgery, Central South University Xiangya School of \\ Medicine Affiliated Haikou Hospital, Haikou, Hainan 570208, P.R. China
}

Received July 16, 2018; Accepted January 24, 2019

DOI: $10.3892 /$ etm.2019.7526

\begin{abstract}
The long non-coding RNA small nucleolar RNA host gene 12 (SNHG12) has recently been reported to have an oncogenic role in gastric cancer (GC), but the molecular mechanisms remain largely elusive. In the present study, it was observed that SNHG12 was significantly upregulated in GC tissues and cell lines. High expression of SNHG12 was associated with GC progression and poor prognosis of patients. Knockdown of SNHG12 markedly inhibited the proliferation and migration of the BGC823 and HGC27 GC cell lines. MicroRNA (miR)-16 was identified as a target of SNHG12, and its expression was negatively regulated by SNHG12 in BGC823 and HGC27 cells. In addition, the expression of miR-16 was significantly decreased in GC tissues and cell lines, and inversely associated with the expression of SNHG12 in GC tissues. Furthermore, knockdown of miR-16 impaired the inhibitory effects on GC cell proliferation and migration induced by downregulation of SNHG12. In conclusion, the present study demonstrates that inhibition of SNHG12 suppresses GC cell proliferation and migration by modulation of miR-16 expression, and thus suggests that the SNHG12/miR-16 interaction may be used as a promising target for GC treatment.
\end{abstract}

\section{Introduction}

Gastric cancer (GC) is one of the most common human cancer types, and the second leading cause of cancer-associated death worldwide, particularly in East Asia $(1,2)$. Although great effort has been made to improve its treatment, GC remains difficult to cure, mainly due to most GC patients presenting with advanced disease and/or metastasis at the

Correspondence to: Dr Guodong Zhao, Department of Gastrointestinal Surgery, Central South University Xiangya School of Medicine Affiliated Haikou Hospital, 43 Renmin Road, Haikou, Hainan 570208, P.R. China

E-mail: guodongzh2018@163.com

Key words: gastric cancer, small nucleolar RNA host gene 12, microRNA-16, proliferation, migration time-point of diagnosis $(3,4)$. Therefore, the elucidation of the exact mechanisms underlying GC growth and metastasis is urgently required.

Long non-coding RNAs (lncRNAs), a class of non-coding RNAs comprising >200 nucleotides, may exert their functions through sponging their target microRNAs (miRs), mRNAs or proteins, and affecting their expression (5-7). In the last decade, accumulating evidence has indicated that lncRNAs have important roles in physiological and pathological processes (8-10). Furthermore, the deregulation of lncRNAs has been implicated in human cancers, including GC $(11,12)$. For instance, the lncRNA $X$ inactive specific transcript (XIST) was reported to be significantly upregulated in GC cells, and to promote GC progression through transforming growth factor- $\beta 1$ via targeting miR-185 (12). In addition, the IncRNA nuclear paraspecle assembly transcript was indicated to regulate GC development through modulating the expression of miR-506 and signal transducer and activator of transcription 3 (13). In addition, the lncRNA small nucleolar RNA host gene (SNHG)20 promotes GC progression by inhibiting $\mathrm{p} 21$ expression and regulating the glycogen synthase kinase (GSK)-3 $\beta / \beta$-catenin signaling pathway (11).

The lncRNA SNHG12 is frequently upregulated in several common human cancer types and promotes tumorigenesis by acting as a sponge for certain miRs $(14,15)$. For instance, SNHG12 is significantly upregulated in osteosarcoma tissues and cell lines, and promotes osteosarcoma cell proliferation, invasion and migration through increasing the expression of angiomotin, as well as Notch2 by sponging miR-195-5p (14,15). Wang et al (16) reported that SNHG12 promotes colorectal cancer cell growth and inhibits cell apoptosis. In addition, C-Myc-induced upregulation of SNHG12 enhanced the proliferation, apoptosis and migration of triple-negative breast cancer cells (17). In addition, upregulation of SNHG12 was identified to contribute to cervical cancer cell proliferation and invasion by acting as a sponge for miR-424-5p (18). Recently, Zhang and Lu (19) reported that SNHG12 has a promoting role in GC by acting as a molecular sponge for miR-320. However, whether SNHG12 also interacts with other miRs in GC cells still remains to be elucidated. Therefore, the present study aimed to explore the regulatory mechanisms of SNHG12 underlying GC cell proliferation and migration. 


\section{Materials and methods}

Tissue samples. The present study was approved by the Medical Ethics Committee of Haikou People's Hospital (Haikou, China) and complied with the Declaration of Helsinki. A total of 56 primary GC tissues as well as their matched adjacent non-tumor tissues were obtained from GC patients treated at Haikou People's Hospital (Haikou, China) between May 2011 and May 2013. The patients included 34 males and 22 females, between 38-77 years old with mean age of 65.6 years old. These GC patients did not receive any radiotherapy or chemotherapy prior to surgical resection. Written informed consent had been obtained from all patients. The tissues were frozen in liquid nitrogen immediately after surgical resection and stored at $-80^{\circ} \mathrm{C}$ until use.

Cell culture. The AGS, HGC27, BGC823 and SGC7901 human GC cell lines and the GES-1 normal gastric mucosa epithelial cell line and were obtained from the Cell Bank of the Chinese Academy of Sciences (Shanghai, China). The cells were cultured in Dulbecco's modified Eagle's medium (DMEM; Thermo Fisher Scientific, Inc., Waltham, MA, USA) with $10 \%$ fetal bovine serum (FBS; Thermo Fisher Scientific, Inc.) at $37^{\circ} \mathrm{C}$ in a humidified atmosphere with $5 \% \mathrm{CO}_{2}$. The cells were harvested during the logarithmic growth phase for use in the subsequent experiments.

Cell transfection. BGC823 and HGC27 cells were transfected with two SNHG12 small interfering (si)RNAs that have different targets (100 $\mu \mathrm{M}$; cat. nos. AM16708 and 1299001), negative control (NC) siRNA (cat. no. 4457289; all Thermo Fisher Scientific, Inc.), pcDNA-SNHG12 expression plasmid (cat. no. E2425; Hunan Nanhua Aishi Pulin Biotechnology; NanHua Bio-medicine Co., Ltd., Changsha, China), a pcDNA3.1 vector (cat. no. V79020), or were co-transfected with SNHG12 siRNA and miR-16 inhibitor (cat. no. 4464084) or SNHG12 siRNA and NC inhibitor (cat. no. AM17010) using Lipofectamine 2000 (all Thermo Fisher Scientific, Inc.) according to the manufacturer's protocol. At $48 \mathrm{~h}$ after transfection, the cells were used for the subsequent experiments.

Reverse transcription-quantitative polymerase chain reaction ( $R T-q P C R)$. TRIzol reagent (Thermo Fisher Scientific, Inc.) was used to extract total RNA from tissues and cells. For detection of SNHG12 expression, $2 \mu \mathrm{g}$ total RNA was used to synthesize complementary DNA using SuperScript III Reverse Transcriptase (Thermo Fisher Scientific, Inc.) according to the manufacturer's protocol. qPCR was then performed using Fast SYBR ${ }^{\text {TM }}$ Green Master Mix (cat. no. 4385610; Applied Biosystems; Thermo Fisher Scientific, Inc.), according to the manufacturer's protocol. For detection of miR-16 expression, the Mir- $\mathrm{X}^{\mathrm{TM}}$ miRNA qRT-PCR SYBR ${ }^{\circledR}$ kit (Clontech Laboratories, Inc., Mountainview, CA, USA) was applied for RT-qPCR according to the manufacturer's protocol. GAPDH and U6 were used as internal references. The reaction conditions were $95^{\circ} \mathrm{C}$ for $3 \mathrm{~min}$, followed by 40 cycles of $95^{\circ} \mathrm{C}$ for $15 \mathrm{sec}$ and $60^{\circ} \mathrm{C}$ for $30 \mathrm{sec}$. The relative expression was analyzed using the $2^{-\Delta \Delta C \mathrm{Cq}}$ method (20).
Cell Counting Kit (CKK)-8 assay. BGC823 and HGC27 cells were re-suspended with DMEM and placed into 96-well plates $\left(5,000\right.$ cells in $200 \mu \mathrm{l}$ per well). After incubation at $37^{\circ} \mathrm{C}$ for 0,24, 48 or $72 \mathrm{~h}, 10 \mu \mathrm{l} \mathrm{CCK}-8$ reagent (Beyotime Institute of Biotechnology, Haimen, China) was added to each well. After incubation at $37^{\circ} \mathrm{C}$ for $2 \mathrm{~h}$, the absorbance at $450 \mathrm{~nm}$ was quantitated using a Synergy ${ }^{\mathrm{TM}}$ LX Multi-Mode microplate reader (BioTek Instruments, Inc., Winooski, VT, USA).

Cell migration assay. A wound healing assay was used to assess cell migration. In brief, transfected BGC823 and HGC27 cells (500,000 cells per well) were seeded into 6-well plates and cultured for $24 \mathrm{~h}$. A wound was scratched in the cell monolayer using a 200- $\mu 1$ pipette tip (cat. no. 94052320; Thermo Fisher Scientific, Inc.). Cells were washed with Dulbecco's PBS (Thermo Fisher Scientific, Inc.) and DMEM was then added to the 6-well plates. Images of the scraped area were captured at 0 and 24 h using an inverted microscope (Olympus, Tokyo, Japan).

Luciferase reporter gene assay. A bioinformatics analysis was performed to determine potential target miRs of SNHG12 by using an online prediction tool (Starbase version 1.0; http://starbase.sysu.edu.cn/mirLncRNA.php). To construct a luciferase reporter vector, the 3'untranslated region (UTR) fragment of SNHG12 containing the putative binding site for miR-16 was amplified by PCR, which was then inserted into the multiple cloning region located downstream of the Renilla translational stop codon in the psi-CHECK2 Luciferase reporter vector (Promega Corp., Madison, WI, USA), named as wild-type (WT) SNHG12 3'UTR. In addition, the mutant (MT) 3'UTR fragment of SNHG12 without the putative binding sites for miR-16 was generated, which was also inserted into the multiple cloning region of psi-CHECK2 Luciferase vector, and named as MT SNHG12 3'UTR. BGC823 and HGC27 cells were co-transfected with $0.5 \mu \mathrm{g}$ WT SNHG12 3'UTR or MT SNHG12 3'UTR and miR-16 mimics or miR-NC using Lipofectamine 2000 according to the manufacturer's protocol. At $48 \mathrm{~h}$ after transfection, a Dual-luciferase Reporter Assay kit (cat. no. E1910; Promega Corp.) was used to examine the luciferase activity according to the manufacturer's protocol.

Statistical analysis. Values are expressed as the mean \pm standard deviation. Student's t-test was used for analyzing the difference between two groups. For comparison of more than two groups, one-way analysis of variance was used followed by Tukey's post-hoc test. The correlation between the SNHG12 and miR-16 expression in GC tissues was analyzed using Pearson's correlation analysis. Kaplan-Meier analysis with a log-rank test was performed to assess patient survival. The chi-square test was employed to analyze the associations between SNHG12 expression and clinicopathological characteristics of GC patients. GraphPad Prism 6.0 software (GraphPad Software Inc., La Jolla, CA, USA) was used to perform statistical analyses. $\mathrm{P}<0.05$ was considered to indicate statistical significance.

\section{Results}

Upregulation of SNHG12 is associated with GC progression. First, the expression of SNHG12 in GC tissues and their 

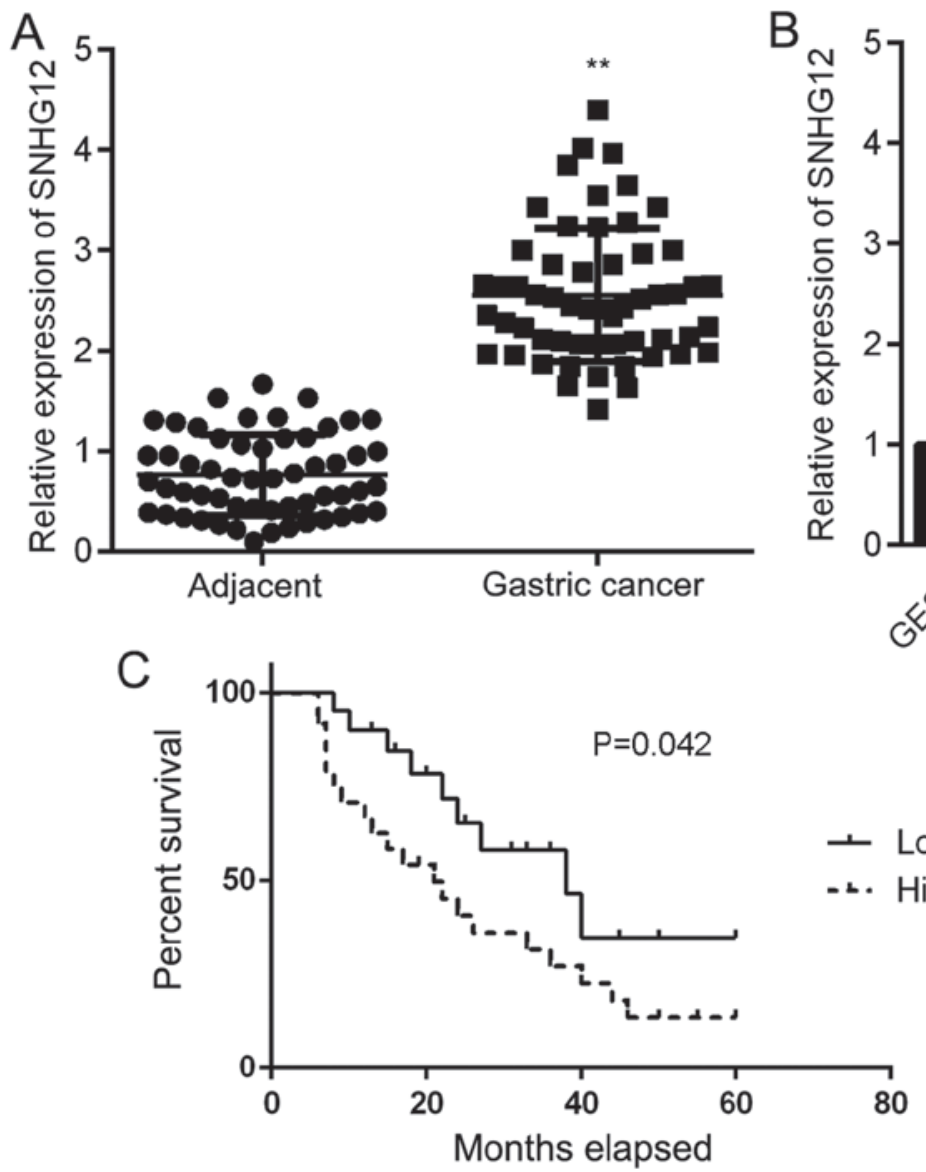

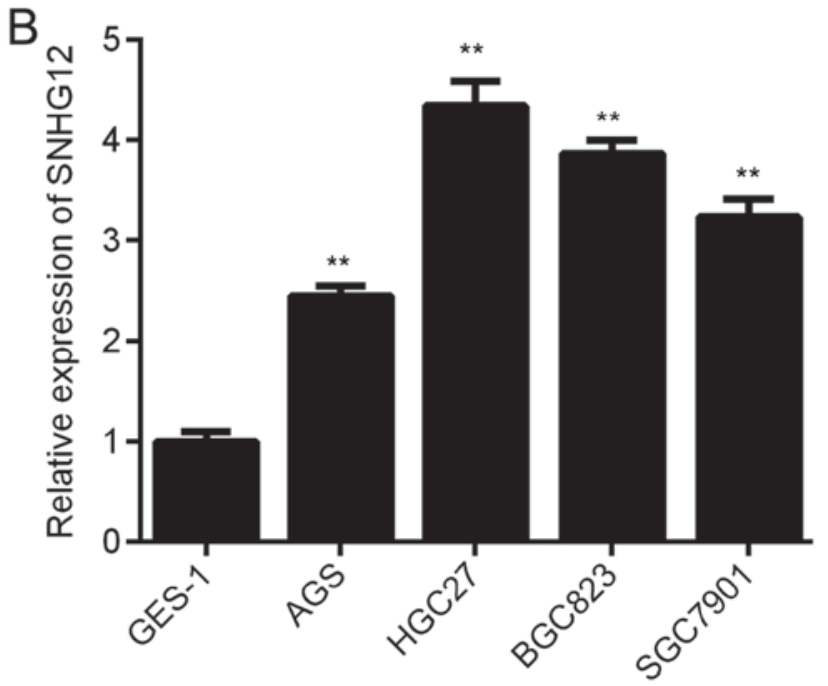

- Low SNHG12 expression

- High SNHG12 expression

Figure 1. Upregulation of SNHG12 is associated with GC progression. (A) SNHG12 is significantly upregulated in GC tissues compared with adjacent non-tumor tissues. (B) SNHG12 is significantly upregulated in GC cell lines compared with the normal gastric mucosa epithelial cell line GES-1. ${ }^{* *} \mathrm{P}<0.01$ vs. adjacent or GES-1. (C) The follow-up time for these patients was 60 months. Kaplan-Meier curves indicating that GC patients with high SNHG12 expression has a shorter survival time when compared with those with low SNHG12 expression. SNHG12, small nucleolar RNA host gene 12; GC, gastric cancer.

matched adjacent normal tissues was examined by using RT-qPCR. As shown in Fig. 1A, the expression of SNHG12 was significantly higher in GC tissues when compared to the adjacent tissues. Consistently, SNHG12 was also upregulated in GC cell lines, including AGS, BGC823 HGC27 and SGC7901, when compared with that in the normal gastric mucosa epithelial cell line GES-1 (Fig. 1B). Thus, SNHG12 was generally upregulated in GC. Subsequently, the clinical significance of SNHG12 expression in GC was assessed. The GC patients were divided into a high and a low SNHG12 expression group, based on its mean expression value in GC tissues. The results suggested that high SNHG12 expression was significantly associated with a larger tumor size, advanced clinical stage and lymph node metastasis (Table I). Furthermore, those patients with high SNHG12 expression had a shorter survival time when compared with those with low SNHG12 expression (Fig. 1C). Therefore, upregulation of SNHG12 is associated with GC progression.

Knockdown of SNHG12 inhibits GC cell proliferation and migration. As SNHG12 was markedly upregulated in GC, the effects of SNHG12 downregulation on the behavior of GC cells were then assessed in vitro. BGC823 and HGC27 cells were selected for the subsequent experiments, as these cell lines had the highest expression levels of SNHG12. Two
SNHG12 siRNAs were used to knock down the expression of SNHG12 in GC cells. As presented in Fig. 2A, transfection with SNHG12 siRNA and siRNA2 significantly reduced the SNHG12 expression levels in BGC823 and HGC27 cells, when compared with those in the NC siRNA group. SNHG12 siRNA was then selected for the subsequent experiments, as it demonstrated a better knockdown efficiency. A CKK-8 assay and a wound healing assay then indicated that knockdown of SNHG12 significantly reduced the proliferation and migration, respectively, of BGC823 and HGC27 cells (Fig. 2B-E). Based on these results, SNHG12 may have a promoting role in $\mathrm{GC}$ growth and metastasis.

SNHG12 directly targets miR-16 in GC cells. A Bioinformatics analysis was performed to determine potential target miRs of SNHG12 by using an online prediction tool. As presented in Fig. 3A, miR-16 was predicted to be a potential target of SNHG12. To verify this prediction, the luciferase reporter plasmids containing the WT and MT miR-16 binding sites in SNHG12 were generated (Fig. 3A). GC cells were first transfected with miR-16 mimics or miR-NC mimics, and it was confirmed that after transfection, the miR-16 levels were significantly upregulated in the miR-16 group compared with those in the miR-NC group (Fig. 3B). Subsequently, a luciferase reporter gene assay was performed to verify the targeting association between 
Table I. Association between SNHG12 expression and clinicopathological characteristics of gastric cancer patients.

\begin{tabular}{|c|c|c|c|c|}
\hline \multirow[b]{2}{*}{ Parameter } & \multirow[b]{2}{*}{ Cases $(n=56)$} & \multicolumn{2}{|c|}{ SNHG12 levels } & \multirow[b]{2}{*}{ P-value } \\
\hline & & Low $(\mathrm{n}=31)$ & High $(n=25)$ & \\
\hline Age (years) & & & & 1.000 \\
\hline$\leq 60$ & 19 & 11 & 8 & \\
\hline$>60$ & 37 & 20 & 17 & \\
\hline Sex & & & & 0.278 \\
\hline Male & 34 & 21 & 13 & \\
\hline Female & 22 & 10 & 12 & \\
\hline Tumor size (cm) & & & & 0.035 \\
\hline$\leq 5$ & 27 & 19 & 8 & \\
\hline$>5$ & 29 & 12 & 17 & \\
\hline Differentiation & & & & 0.057 \\
\hline Well and moderately & 33 & 22 & 11 & \\
\hline Poor & 23 & 9 & 14 & \\
\hline Node metastasis & & & & 0.006 \\
\hline Present & 48 & 23 & 25 & \\
\hline Absent & 8 & 8 & 0 & \\
\hline Clinical stage & & & & 0.045 \\
\hline I-II & 17 & 13 & 4 & \\
\hline III-IV & 39 & 18 & 21 & \\
\hline
\end{tabular}

SNHG12, small nucleolar RNA host gene 12 .

miR-16 and SNHG12. The results indicated that overexpression of miR-16 markedly inhibited the luciferase activity of WT SNHG12 in BGC823 and HGC27 cells, but had no effect on the luciferase activity of MT SNHG12 (Fig. 3C and D), suggesting that SNHG12 directly targets miR-16 in GC cells. The effects of SNHG12 on the expression of miR-16 in GC cells was then assessed. As presented in Fig. 3E, downregulation of SNHG12 significantly increased the miR-16 expression in BGC823 and HGC27 cells, suggesting that SNHG12 negatively regulates the expression of miR-16 in GC cells. To further confirm these results, BGC823 and HGC27 cells were transfected with SNHG12 expression plasmid to increase its expression. As presented in Fig. 3F, transfection with SNHG12 expression plasmid significantly enhanced its expression in GC cells, when compared with that in the blank group. Indeed, upregulation of SNHG12 led to a significant reduction in the expression of miR-16 in GC cells (Fig. 3G). Thus, SNHG12 negatively regulates the miR-16 expression in GC cells.

miR-16 is downregulated in GC tissues and cell lines. The expression of miR-16 in GC tissues and cell lines was then assessed using RT-qPCR. The results indicated that miR-16 was markedly downregulated in GC tissues compared with that in adjacent non-tumor tissues (Fig. 4A). In addition, it was also downregulated in GC cell lines compared with that in GES-1 cells (Fig. 4B). Therefore, miR-16 is downregulated in GC. Subsequently, a Pearson correlation analysis was performed to determine the correlation between SNHG12 and miR-16 expression in GC tissues. As provided in Fig. 4C, an inverse association was identified between SNHG12 and miR-16 expression in GC tissues, suggesting that upregulation of SNHG12 may contribute to the downregulation of miR-16 in GC tissues.

Knockdown of miR-16 impairs the suppressive effects of SNHG12 downregulation on GC cell proliferation and migration. Based on the above results, it was speculated that miR-16 may be important for the biological effects of SNHG12 on GC cells. To test this hypothesis, GC cells were transfected with NC inhibitor or miR-16 inhibitor. After transfection, the miR-16 levels were significantly reduced in the anti-miR-16 group compared with those in the anti-NC group (Fig. 5A). Subsequently, the SNHG12 siRNA-transfected GC cells were transfected with miR-16 inhibitor or NC inhibitor. As provided in Fig. 5B, the miR-16 levels were markedly reduced in the siSNHG12+anti-miR-16 group compared with those in the siSNHG12+anti-NC group. A CKK-8 assay and a wound healing assay were then performed. As indicated in Fig. 5C-F, the proliferation and migration of GC cells were significantly upregulated in the siSNHG12+anti-miR-16 group when compared with those in the siSNHG12+anti-NC group. These results suggest that inhibition of miR-16 impairs the suppressive effects of SNHG12 downregulation on GC cell proliferation and migration.

\section{Discussion}

The mechanisms of the effect of SNHG12 to promote GC progression remains largely elusive. The present study 
A
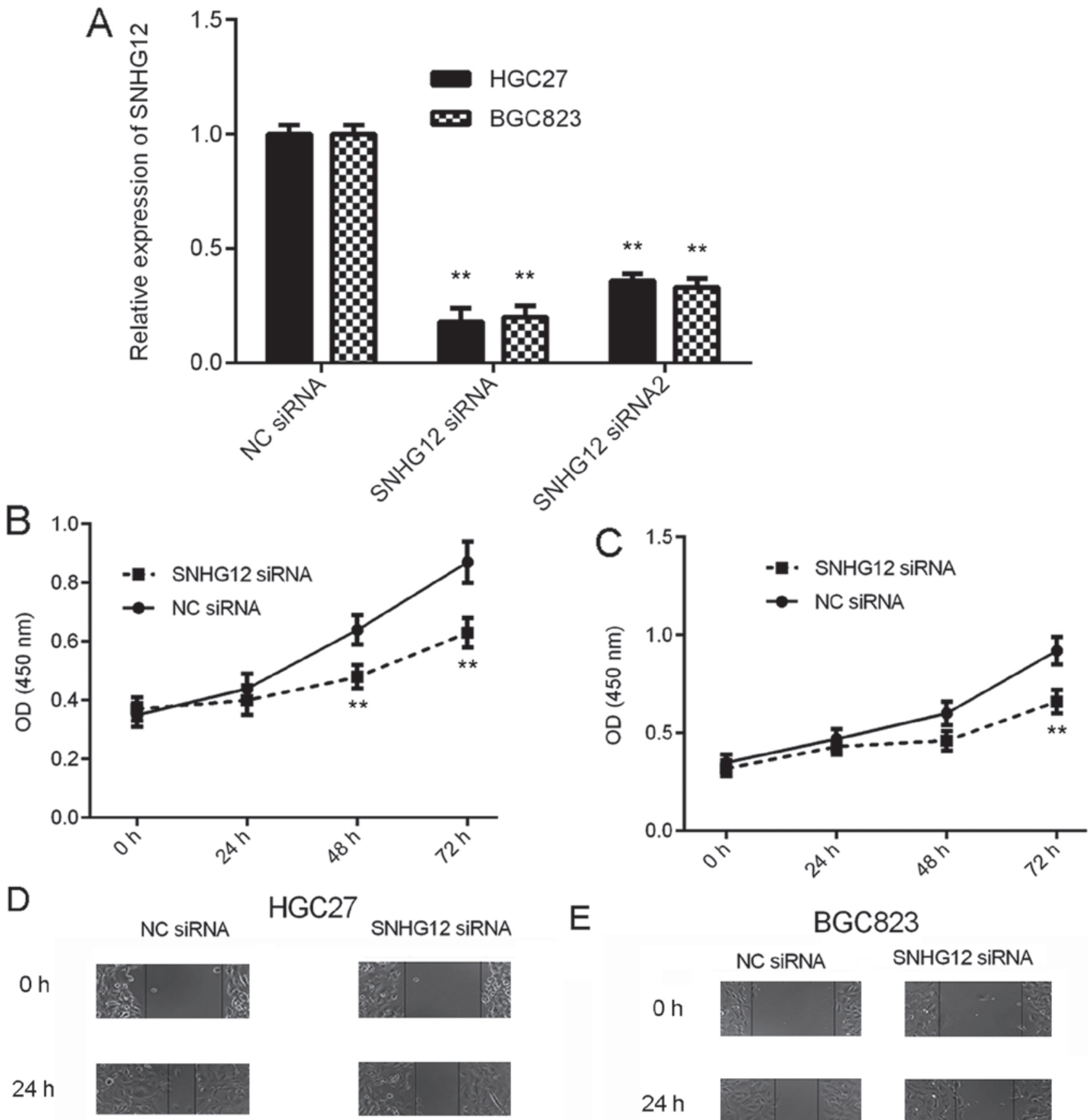

$E$

BGC823
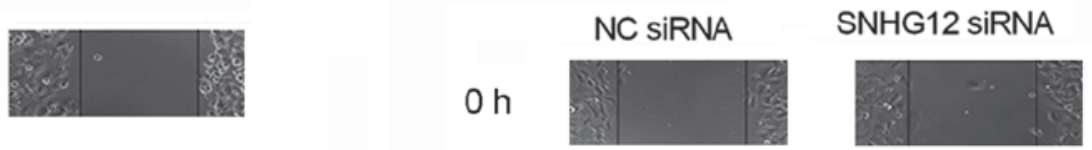

$24 \mathrm{~h}$
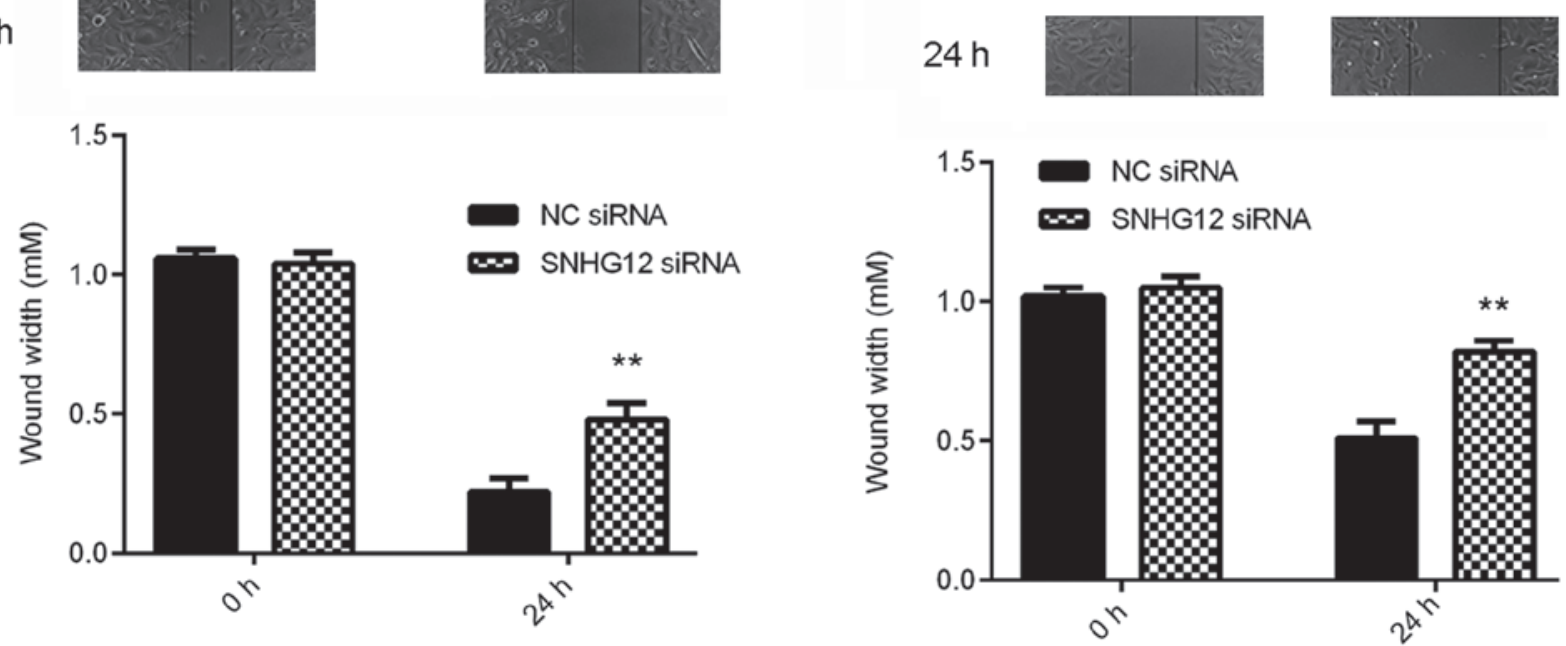

Figure 2. Knockdown of SNHG12 inhibits gastric cancer cell proliferation and migration. BGC823 and HGC27 cells were transfected with SNHG12 siRNA, SNHG12 siRNA2 or NC siRNA. (A) Reverse transcription-quantitative polymerase chain reaction analysis was used to determine the expression of SNHG12. (B and C) A Cell Counting Kit-8 assay was performed to study cell proliferation and (D and E) a wound healing assay was performed to assess cell migration. Magnification, $x 40 .{ }^{* *} \mathrm{P}<0.01$ vs. NC siRNA. SNHG12, small nucleolar RNA host gene 12; siRNA, small interfering RNA; NC, negative control; OD, optical density. 


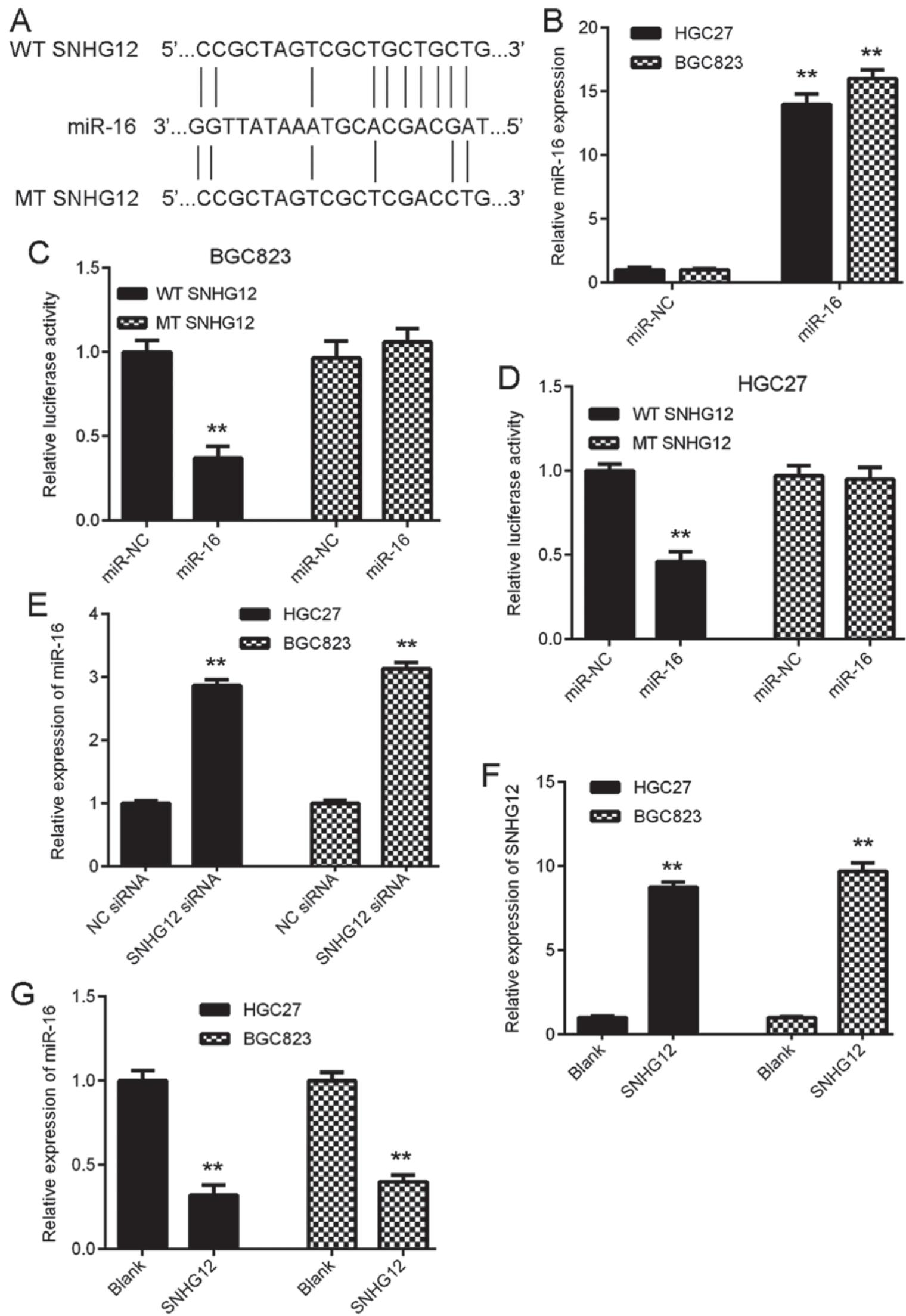

Figure 3. SNHG12 directly targets miR-16 in GC cells. (A) miR-16 was predicted as a potential target of SNHG12, and luciferase reporter plasmids containing the WT and MT miR-16 binding sites in SNHG12 were generated. (B) RT-qPCR analysis was performed to examine the miR-16 expression in GC cells after transfection with miR-NC or miR-16 mimics. (C and D) The luciferase reporter gene assay indicated that miR-16 mimics markedly inhibited the luciferase activity of the reporter plasmid containing WT SNHG12 in BGC823 and HGC27 cells, but had no effect on the luciferase activity of the reporter plasmid containing MT SNHG12. (E) Transfection with SNHG12 siRNA caused a significant upregulation of the expression of miR-16 in BGC823 and HGC27 cells. (F and G) BGC823 and HGC27 cells were transfected with SNHG12 expression plasmid or blank vector. RT-qPCR was performed to examine the expression of (F) SNHG12 and (G) miR-16. ${ }^{* *} \mathrm{P}<0.01$ vs. miR-NC, NC siRNA or Blank. SNHG12, small nucleolar RNA host gene 12; WT, wild-type; MT, mutated type; miR, microRNA; siRNA, small interfering RNA; NC, negative control; GC, gastric cancer; RT-qPCR, reverse transcription-quantitative polymerase chain reaction. 
A

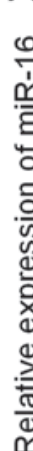

일

1

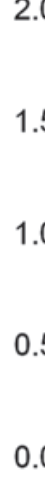

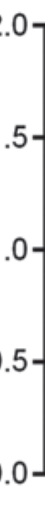

10

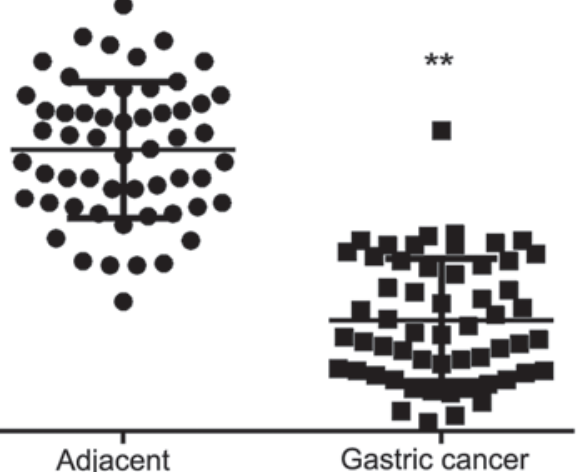

B

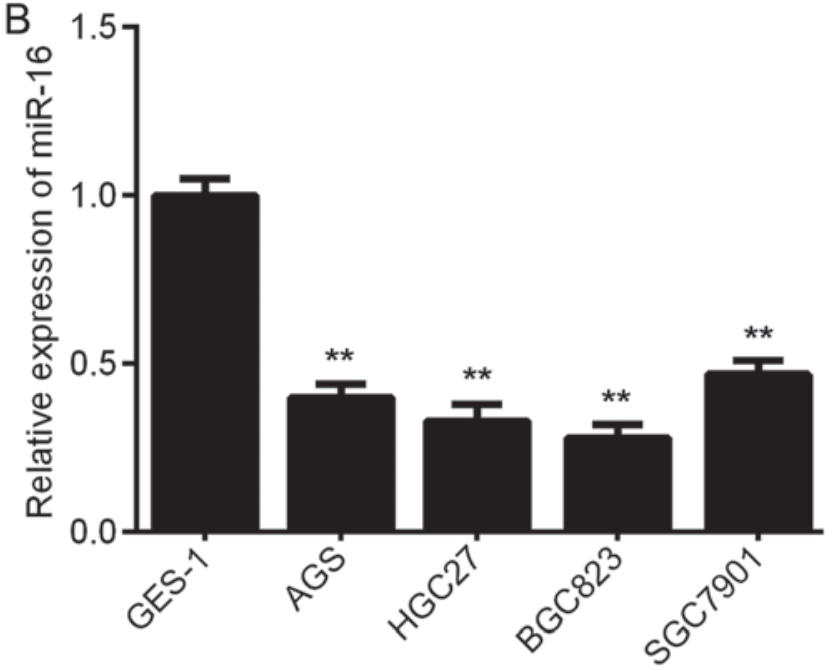

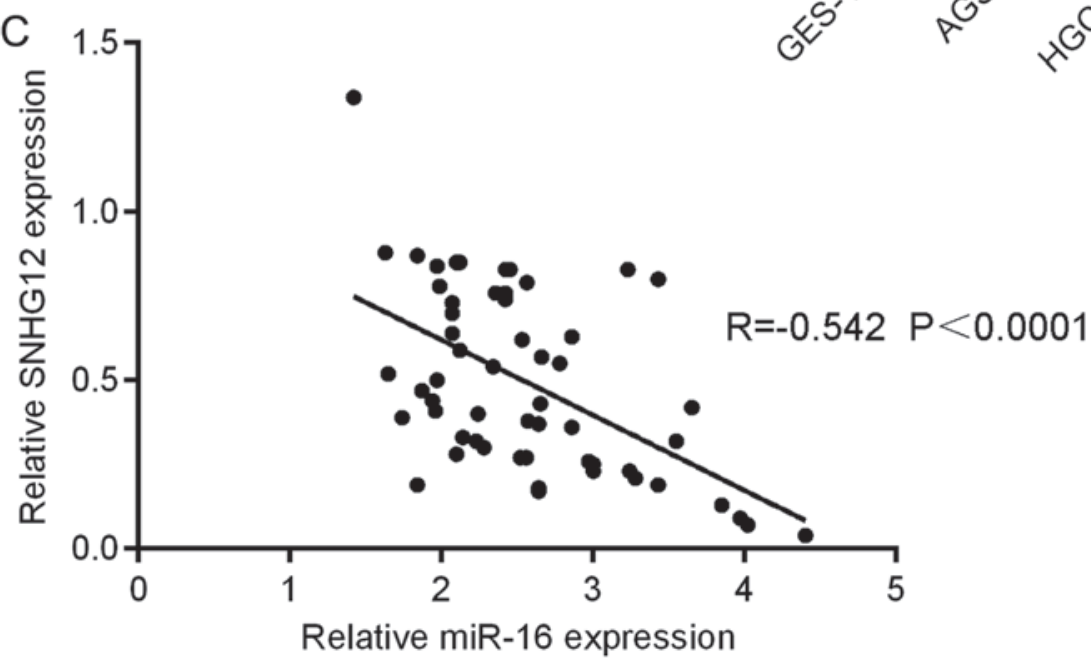

Figure 4. miR-16 is downregulated in GC tissues and cell lines. (A) RT-qPCR analysis indicated that miR-16 was markedly downregulated in GC tissues compared to adjacent non-tumor tissues. ${ }^{* * *} \mathrm{P}<0.01$ vs. Adjacent. (B) RT-qPCR analysis indicated that miR-16 was markedly downregulated in GC cell lines compared with that in the normal gastric mucosa epithelial cell line GES-1. ${ }^{* *} \mathrm{P}<0.01$ vs. GES-1. (C) An inverse correlation between the SNHG12 and miR-16 expression levels was determined in GC tissues. GC, gastric cancer; SNHG12, small nucleolar RNA host gene 12; RT-qPCR, reverse transcription-quantitative polymerase chain reaction; $\mathrm{miR}$, microRNA.

reported that SNHG12 was significantly upregulated in GC tissues and cell lines, and high SNHG12 expression was associated with GC progression and poor prognosis. Knockdown of SNHG12 markedly inhibited the proliferation and migration of the BGC823 and HGC27 GC cell lines. miR-16 was identified as a target of SNHG12, and its expression was negatively regulated by SNHG12 in BGC823 and HGC27 cells. Furthermore, the expression of miR-16 was significantly decreased in GC tissues and cell lines, and inversely associated with the expression of SNHG12 in GC tissues. In addition, knockdown of miR-16 impaired the inhibitory effects on GC cell proliferation and migration induced by SNHG12 knockdown.

SNHG12 has been reported to be frequently upregulated and to have a promoting role in various common human cancer types $(14,15,18)$. For instance, SNHG12 was significantly upregulated in liver cancer tissues compared with that in the adjacent normal tissues, and knockdown of SNHG12 effectively reduced cancer cell proliferation, migration and invasion (21). Ding et al (22) reported that SNHG12 exerted promoting effects on the proliferation, migration and invasion of papillary thyroid carcinoma cells through regulating the Wnt/ $\beta$-catenin signaling pathway. The results of the present study indicated that SNHG12 was markedly upregulated in GC tissues and cells compared with that in adjacent normal tissues and GES-1 cells, respectively. It was further observed that the expression of SNHG12 was associated with a larger tumor size, tumor metastasis and advanced clinical stage, as well as poor prognosis of GC patients; this was consistent with the results of a previous study by Zhang and $\mathrm{Lu}$ (19), which also demonstrated that inhibition of SNHG12 suppressed the proliferation, colony formation and invasion of GC SGC-7901 and AGS cells. In the present study, in vitro experiments revealed that knockdown of SNHG12 inhibited the proliferation and migration of the BGC823 and $\mathrm{HGC} 27$ GC cell lines. These present results expand the understanding of the function of SNHG12 in GC cells.

It has been well established that lncR NAs negatively regulate the expression of miRs through acting as sponges for them in GC cells (7). For instance, the IncRNA SNHG20 promotes GC progression by inhibition of $\mathrm{p} 21$ expression and regulating the GSK-3 $\beta / \beta$-catenin signaling pathway (11). The lncRNA XIST promotes GC progression via targeting miR-185 (12). Thus, a Bioinformatics analysis and a luciferase reporter gene assay were then performed to study the potential target miRs of SNGH12, and the results 
A

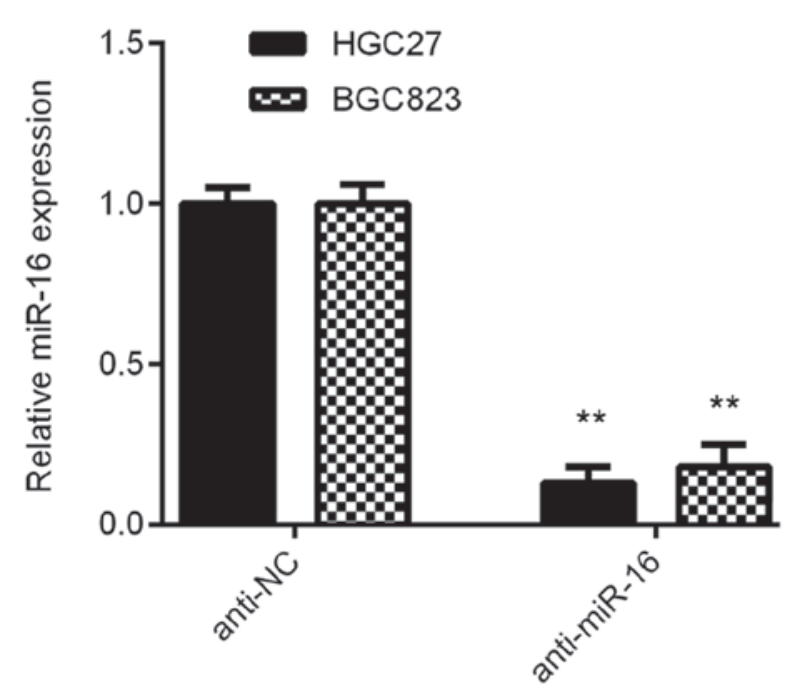

C

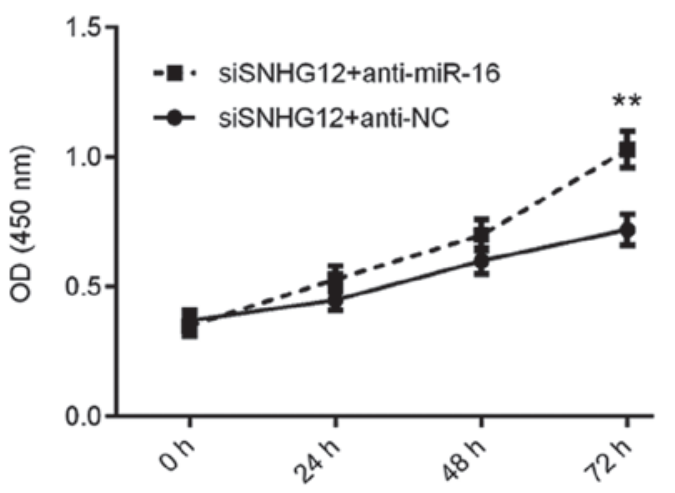

E

$\mathrm{Oh}$

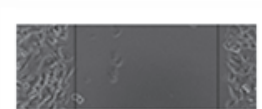

HGC27

siSNHG12+anti-miR-16

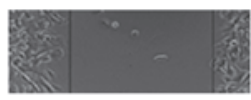

$24 \mathrm{~h}$
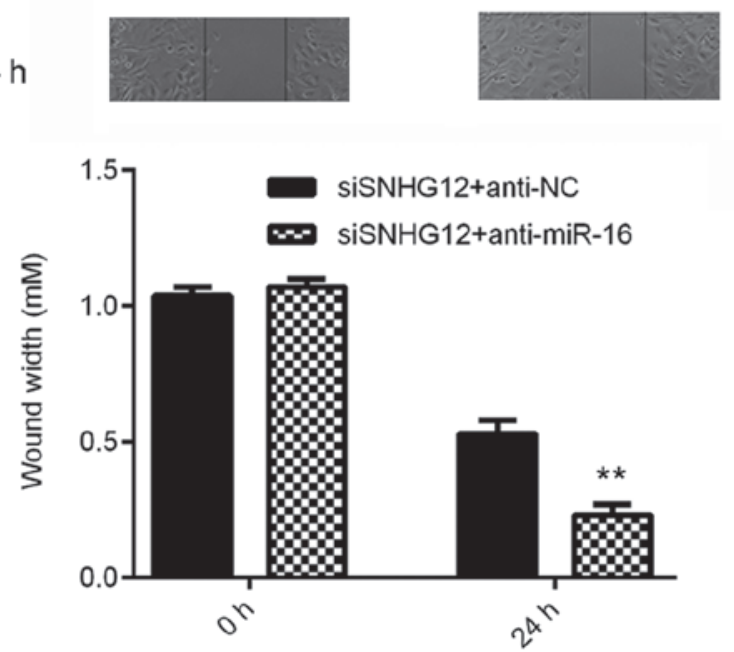

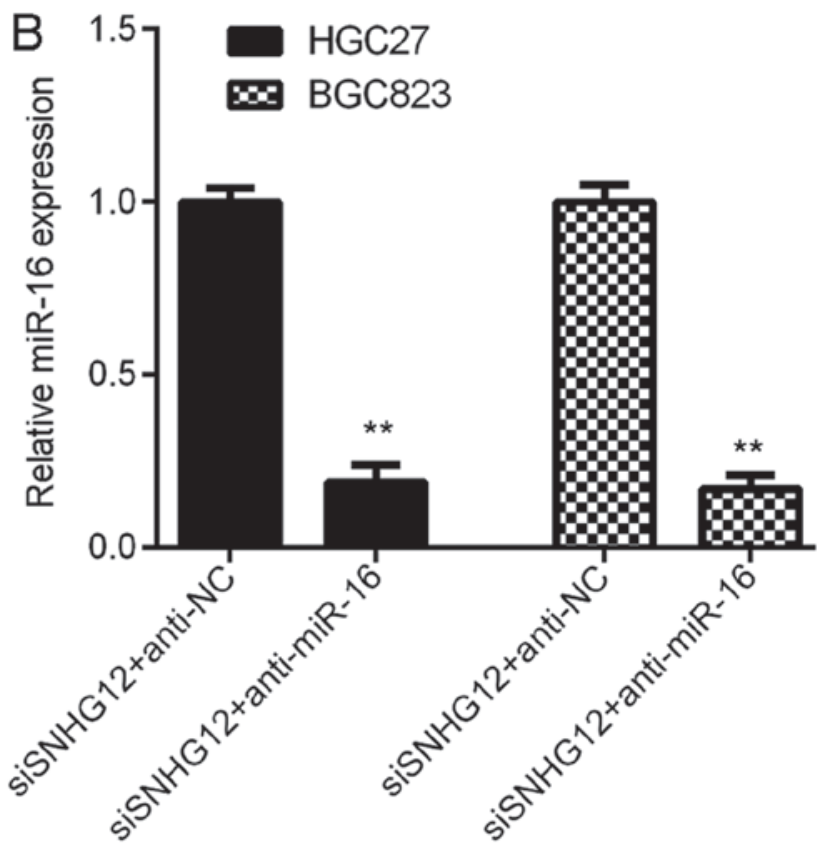

D

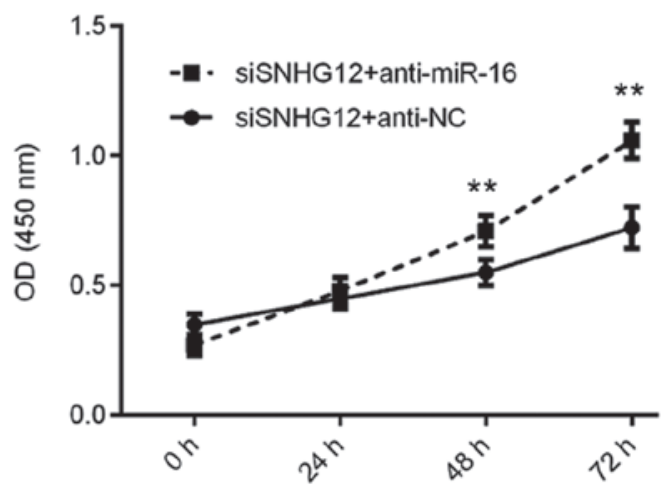

F

BGC823

$\mathrm{Oh}$

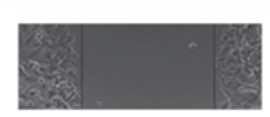

siSNHG12+anti-miR-16

$24 \mathrm{~h}$
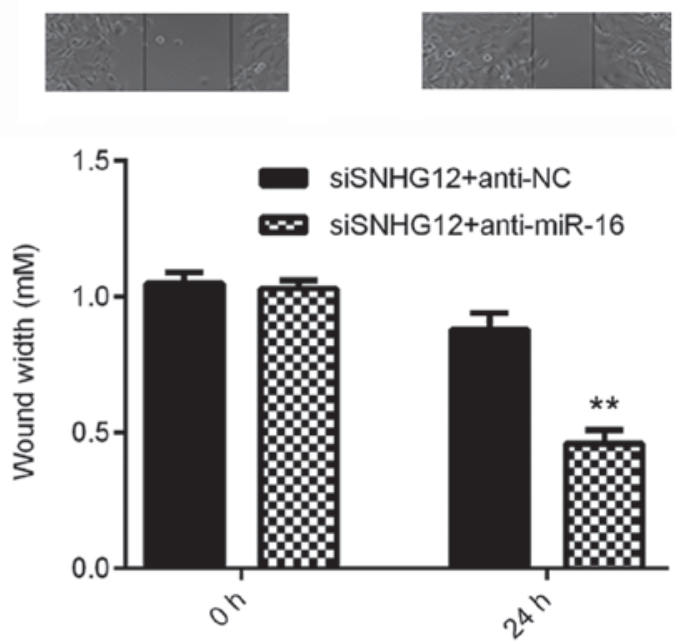

Figure 5. Knockdown of miR-16 impairs the suppressive effects of SNHG12 downregulation on GC cell proliferation and migration. (A) RT-qPCR was used to examine the miR-16 expression in GC cells after transfection with miR-16 inhibitor or NC inhibitor. (B-F) The siSNHG12-transfected GC cells were transfected with miR-16 inhibitor or NC inhibitor. (B) RT-qPCR was used to examine the expression of miR-16. (C and D) A Cell Counting Kit-8 assay was performed to study cell proliferation and $(\mathrm{E}$ and $\mathrm{F})$ a wound healing assay was performed to assess cell migration. Magnification, $\mathrm{x} 40$. ${ }^{* * *} \mathrm{P}<0.01 \mathrm{vs}$. siSNHG12+anti-NC. SNHG12, small nucleolar RNA host gene 12; miR, microRNA; NC, negative control; siRNA, small interfering RNA; siSNHG12, siRNA specific for SNHG12; GC, gastric cancer; RT-qPCR, reverse transcription-quantitative polymerase chain reaction; OD, optical density. 
demonstrated that miR-16 was a potential target of SNHG12. A further experiment confirmed that SNHG12 negatively regulated the expression of miR-16 in BGC823 and HGC27 cells. Subsequently, the correlation between the expression of miR-16 and SNHG12 in GC tissues was examined. The results indicated that the expression of miR-16 was significantly reduced in GC tissues and cell lines, and inversely correlated with the expression of SNHG12 in GC tissues, suggesting that the increased expression of SNHG12 may contribute to the reduced expression of miR-16 in GC.

miR-16 has been reported to have a tumor suppressive role in several common cancer types, including GC (23-25). For instance, miR-16 may inhibit glioma cell growth and invasion through suppressing B-cell lymphoma 2 as well as the nuclear factor $-\kappa \mathrm{B} 1 /$ matrix metallopeptidase 9 signaling pathway (23). In GC, high expression of miR-16 predicates a favorable prognosis for patients (24). Furthermore, Wang et al (25) reported that miR-16 negatively regulated Twist1 to repress GC cell invasion and metastasis. In addition, Li et al (26) reported that overexpression of miR-16 significantly suppressed GC cell proliferation and migration by inhibition of the hepatocyte growth factor/c-Met pathway. However, the molecular mechanisms by which miR-16 regulates $\mathrm{GC}$ cell proliferation and migration still remain to be fully elucidated. The results of the present study indicated that knockdown of miR-16 impaired the suppressive effects on GC cell proliferation and migration induced by SNHG12 silencing, suggesting that miR-16 is involved in the SNHG12-induced effects on GC cells. In addition to miR-16, several other miRs targeted by SNGH12 have also been identified, including miR-125 (27), miR-138 (28), miR-150 (29), miR-181 (30), miR-195 (15), miR-199 (31), miR-101 (32), miR-320 (19) and miR-195 (21). Therefore, the present study expands the current knowledge of regulatory SNHG12/miR interactions in human cancers.

In conclusion, the present study demonstrated that inhibition of SNHG12 suppresses GC cell proliferation and migration by modulation of miR-16 expression, and thus suggests that the SNHG12/miR-16 interaction may be used as a promising target for GC treatment.

\section{Acknowledgements}

Not applicable.

\section{Funding}

No funding received.

\section{Availability of data and materials}

All data generated or analyzed during the present study are included in this published article.

\section{Authors' contributions}

BP collected clinical tissues. CW performed clinical experiments. GZ, SW and XL performed the in-vitro experiments and statistical analysis. GZ designed the study and wrote the manuscript.

\section{Ethics approval and consent to participate}

The present study was approved by the Ethics Committee of Haikou People's Hospital (Haikou, China) and written informed consent had been obtained from all subjects.

\section{Patient consent for publication}

Not applicable.

\section{Competing interests}

The authors declare that they have no competing interests.

\section{References}

1. Siegel RL, Miller KD and Jemal A: Cancer statistics, 2015. CA Cancer J Clin 65: 5-29, 2015.

2. Torre LA, Bray F, Siegel RL, Ferlay J, Lortet-Tieulent J and Jemal A: Global cancer statistics, 2012. CA Cancer J Clin 62: 87-108, 2015.

3. Cheng XJ, Lin JC and Tu SP: Etiology and prevention of gastric cancer. Gastrointest Tumors 3: 25-36, 2016.

4. Thiel A and Ristimäki A: Targeted therapy in gastric cancer. APMIS 123: 365-372, 2015.

5. An J, Lv W and Zhang Y: LncRNA NEAT1 contributes to paclitaxel resistance of ovarian cancer cells by regulating ZEB1 expression via miR-194. Onco Targets Ther 10: 5377-5390, 2017.

6. Zhou Y, Meng X, Chen S, Li W, Li D, Singer R and Gu W: IMP1 regulates UCA1-mediated cell invasion through facilitating UCA1 decay and decreasing the sponge effect of UCA1 for miR-122-5p. Breast Cancer Res 20: 32, 2018.

7. Zhou Y, Chen Y, Ding W, Hua Z, Wang L, Zhu Y, Qian H and Dai T: LncRNA UCA1 impacts cell proliferation, invasion, and migration of pancreatic cancer through regulating miR-96/FOXO3. IUBMB Life 70: 276-290, 2018.

8. Zhang Y, Qian W, Feng F, Cao Q, Li Y, Hou Y, Zhang L and Fan J: Upregulated lncRNA CASC2 may inhibit malignant melanoma development through regulating miR-18a-5p/RUNX1. Oncol Res 27: 371-377, 2019.

9. Yang C, Wu K, Wang S and Wei G: Long non-coding RNA XIST promotes osteosarcoma progression by targeting YAP via miR-195-5p. J Cell Biochem 119: 5646-5656, 2018.

10. Ruan X: Long noncoding RNA central of glucose homeostasis. J Cell Biochem 117: 1061-1065, 2016.

11. Liu J, Liu L, Wan JX and Song Y: Long noncoding RNA SNHG20 promotes gastric cancer progression by inhibiting p21 expression and regulating the GSK-3 $\beta / \beta$-catenin signaling pathway. Oncotarget 8: 80700-80708, 2017.

12. Zhang Q, Chen B, Liu P and Yang J: XIST promotes gastric cancer (GC) progression through TGF- $\beta 1$ via targeting miR-185. J Cell Biochem 119: 2787-2796, 2018.

13. Tan HY, Wang C, Liu G and Zhou X: Long noncoding RNA NEAT1-modualted miR-506 regulates gastric cancer development through targeting STAT3. J Cell Biochem 120: 4827-4836, 2019.

14. Ruan W, Wang P, Feng S, Xue Y and Li Y: Long non-coding RNA small nucleolar RNA host gene 12 (SNHG12) promotes cell proliferation and migration by upregulating angiomotin gene expression in human osteosarcoma cells. Tumour Biol 37: 4065-4073, 2016.

15. Zhou S, Yu L, Xiong M and Dai G: LncRNA SNHG12 promotes tumorigenesis and metastasis in osteosarcoma by upregulating Notch 2 by sponging miR-195-5p. Biochem Biophys Res Commun 495: 1822-1832, 2018.

16. Wang JZ, Xu CL, Wu H and Shen SJ: LncRNA SNHG12 promotes cell growth and inhibits cell apoptosis in colorectal cancer cells. Braz J Med Biol Res 50: e6079, 2017.

17. Wang O, Yang F, Liu Y, Lv L, Ma R, Chen C, Wang J, Tan Q, Cheng Y, Xia E, et al: C-MYC-induced upregulation of lncRNA SNHG12 regulates cell proliferation, apoptosis and migration in triple-negative breast cancer. Am J Transl Res 9: 533-545, 2017. 
18. Dong J, Wang Q, Li L and Xiao-Jin Z: Upregulation of long non-coding RNA small nucleolar RNA host gene 12 contributes to cell growth and invasion in cervical cancer by acting as a sponge for MiR-424-5p. Cell Physiol Biochem 45: 2086-2094, 2018.

19. Zhang $\mathrm{H}$ and Lu W: LncRNA SNHG12 regulates gastric cancer progression by acting as a molecular sponge of miR320. Mol Med Rep 17: 2743-2749, 2018.

20. Livak KJ and Schmittgen TD: Analysis of relative gene expression data using real-time quantitative PCR and the 2(-Delta Delta C(T)) method. Methods 25: 402-408, 2001.

21. Lan T, Ma W, Hong Z, Wu L, Chen X and Yuan Y: Long non-coding RNA small nucleolar RNA host gene 12 (SNHG12) promotes tumorigenesis and metastasis by targeting miR-199a/b-5p in hepatocellular carcinoma. J Exp Clin Cancer Res 36: 11, 2017.

22. Ding S, Qu W, Jiao Y, Zhang J, Zhang C and Dang S: LncRNA SNHG12 promotes the proliferation and metastasis of papillary thyroid carcinoma cells through regulating wnt $/ \beta$-catenin signaling pathway. Cancer Biomark 22: 217-226, 2018.

23. Yang TQ, Lu XJ, Wu TF, Ding DD, Zhao ZH, Chen GL, Xie XS, Li B, Wei YX, Guo LC, et al: MicroRNA-16 inhibits glioma cell growth and invasion through suppression of BCL2 and the nuclear factor-kB1/MMP9 signaling pathway. Cancer Sci 105: 265-271, 2014

24. Ren C, Chen H,Han C, Fu D, Wang D and Shen M: High expression of miR-16 and miR-451 predicating better prognosis in patients with gastric cancer. J Cancer Res Clin Oncol 142: 2489-2496, 2016.

25. Wang T, Hou J, Li Z, Zheng Z, Wei J, Song D, Hu T, Wu Q, Yang JY and Cai JC: miR-15a-3p and miR-16-1-3p negatively regulate twist1 to repress gastric cancer cell invasion and metastasis. Int J Biol Sci 13: 122-134, 2017.
26. Li S, Zhang H, Wang X, Qu Y, Duan J, Liu R, Deng T, Ning T, Zhang L, Bai M, et al: Direct targeting of HGF by miR-16 regulates proliferation and migration in gastric cancer. Tumour Biol 37: 15175-15183, 2016.

27. Jin XJ, Chen XJ, Zhang ZF, Hu WS, Ou RY, Li S, Xue JS, Chen LL, Hu Y and Zhu H: Long noncoding RNA SNHG12 promotes the progression of cervical cancer via modulating miR-125b/STAT3 axis. J Cell Physiol 234: 6624-6632, 2019.

28. Wang X, Qi G, Zhang J, Wu J, Zhou N, Li L and Ma J: Knockdown of long noncoding RNA small nucleolar RNA host gene 12 inhibits cell growth and induces apoptosis by upregulating miR-138 in nonsmall cell lung cancer. DNA Cell Biol 36: 892-900, 2017.

29. Zhao M, Wang J, Xi X, Tan N and Zhang L: SNHG12 promotes angiogenesis following ischemic stroke via regulating miR-150/VEGF pathway. Neuroscience 390: 231-240, 2018.

30. Wang P, Chen D, Ma H and Li Y: LncRNA SNHG12 contributes to multidrug resistance through activating the MAPK/Slug pathway by sponging miR-181a in non-small cell lung cancer. Oncotarget 8: 84086-84101, 2017.

31. Yin WL, Yin WG, Huang BS and Wu LX: LncRNA SNHG12 inhibits miR-199a to upregulate SIRT1 to attenuate cerebral ischemia/reperfusion injury through activating AMPK signaling pathway. Neurosci Lett 690: 188-195, 2019.

32. Sun Y, Liu J, Chu L, Yang W, Liu H, Li C and Yang J: Long noncoding RNA SNHG12 facilitates the tumorigenesis of glioma through miR-101-3p/FOXP1 axis. Gene 676: 315-321, 2018.

This work is licensed under a Creative Commons Attribution-NonCommercial-NoDerivatives 4.0 International (CC BY-NC-ND 4.0) License. 